\title{
Maternal Dietary Leucine Supplementation Affects Apparent Lactational Activity and Pup Development in Rats
}

\author{
Tina L. JoE and Ameae M. WALKer \\ Division of Biomedical Sciences, University of California, \\ Riverside, CA 92521-0121, USA
}

(Received July 23, 1990)

\begin{abstract}
Summary Previous work from this laboratory has shown mildly elevated dietary leucine to alter the rhythm of prolactin secretion and to interfere with normal estrous cycling in the intact female rat. In this study, we have determined whether these observed effects on prolactin and cycling in turn affected fertility, gestation, mammary gland development or mammary gland morphology during lactation. Groups of control and leucine-supplemented mothers were mated with tested male breeders and a daily record was kept of pup births, pup deaths and general health and development of the pups. The day after weaning, mammary tissue was removed from dams in both groups and prepared for histological examination. In light of the previous results showing estrous cycle interruption and altered prolactin secretion, we report what was a surprising lack of effect of leucine supplementation of fertility, maintenance of gestation or overall development of the mammary glands in this species. Interestingly, however, dietary leucine supplementation was found to markedly after the histological appearance of lactating mammary tissue and to cause a significant increase in the number of litters that contained notably underdeveloped pups.
\end{abstract}

Key Words dietary leucine, prolactin, fertility, gestation, pup development, mammary gland

Previous work from this laboratory (1) showed that mildly elevated dietary leucine changed the rhythm of prolactin secretion during the estrous cycle of rats and abolished normal cycling in these animals. Work by Cooper and Linnoila had also shown an effect of L-tyrosine and L-leucine on cycling in older rats (2). In this study we attempted to determine whether these alterations in prolactin secretion and the estrus cycle would, as one might expect, have any effect on fertility, maintenance of gestation or subsequent lactation. We report no effect of increased dietary leucine on fertility or gestation, but a clear effect on the morphology and implied level of activity of the lactating mammary gland. 


\section{MATERIALS AND METHODS}

Animals. All of the rats were Sprague-Dawley-derived (Simonson-Gilroy, CA) and were shipped directly from the breeder just prior to each experiment. The females weighed between 220 and $225 \mathrm{~g}$ and the males between 350 and $375 \mathrm{~g}$. The males were sold as tested breeders. Upon arrival, the animals adjusted to their new quarters for a period of 7-10 days. All females were initially housed in groups of three, and six control females and nine experimental females were used for each of the three trials. The males were housed separately and individually until breeding was initiated. All animals were given free access to drinking water and to rat chow (Ralston Purina, Richmond, IN) and were subject to constant light/dark cycles (12 $\mathrm{h}$ light $/ 12 \mathrm{~h}$ dark). The animals were disturbed as little as possible throughout the experiment in order to avoid stress effects on pregnancy or pup nurturing.

Administration of leucine. For each experiment, the drinking water of nine of the 15 females was exchanged for $0.5 \%$ leucine (Sigma Chemical, St. Louis, MO) in tap water on day 0 and the leucine water was subsequently refreshed at 2-day intervals. We have previously observed no difference in the volume of leucine water versus control water consumed (1). Leucine at $0.5 \%$ is the level previously demonstrated to produce changes in prolactin secretion and estrous cycling. It was originally chosen to be well within the low physiological range, to have only a mild flavor so as not to alter fluid consumption and to be one-tenth the level shown by Tannous et al. (3) to result in growth depression.

Breeding. On day 7, the male rats (one per group of 3 females) were introduced into the cages. The rats were allowed to breed for a period of 7 (trials 1 and 2) or 10 (trial 3) days, after which time the males were removed and the females were moved to individual cages. During breeding the males drank the same water as their mates. Day 1 of pregnancy was defined as the day a vaginal plug was observed.

Monitoring of pups. A daily record of pup births, pup deaths and general health and behavior of pups was kept from the day of the first birth to delayed weaning at 28 days of age. Special note was made of hair growth as an indicator of pup development.

Preparation of mammary tissue for light microscopy. The day after weaning, mammary tissue associated with the most posterior of the abdomino-inguinal nipples was removed from control and leucine-supplemented mothers with equivalent litter sizes, fixed in Karnovsky's fixative (4) overnight and then dehydrated in an ethanol series to $95 \%$. The tissue was unfiltrated over the next night with glycol methacrylate (Polysciences, Warrington, PA) and polymerized under vacuum for $3 \mathrm{~h}$ the following morning. The blocks were cut on an LKB ultratome $\mathrm{V}$ and $5 \mu \mathrm{m}$ sections were stained with hematoxylin and eosin before photomicrography using a Nikon microphot-FX microscope and Kodak Tech-pan film (Eastman Kodak, Rochester, NY) at ASA 50. 
Statistical analysis. For each parameter documented, the significance of its difference from the control was tested by Student's $t$-test. Each litter was considered an experimental unit and hence the $t$ test and not chi square test of independence was appropriate for such analysis. Table 2 gives the number of degrees of freedom used during calculations of significance.

\section{RESULTS}

As summarized in Table 1, an increase in dietary leucine to a level previously shown to affect the rhythm of prolactin secretion and to abolish normal estrous cycling in otherwise healthy adult female rats, had no apparent effect on fertility as judged by the number of females that did not produce a litter, the number of pups per litter, or by any delay in the birth of the litters.

The most easily observed effect of leucine supplementation was on pup development as illustrated in Table 2. In order to establish some quantitative measure of pup development, underdeveloped pups were defined as having a week or more's delay in hair growth by comparison to their most advanced siblings. Using this criterion, leucine supplementation doubled the number of litters containing underdeveloped pups. When expressed as a percentage of the number of pups per litter, the occurrence of underdeveloped pups (runts) in the leucinesupplemented litters was threefold the occurrence in the control litters.

Although the criterion of developmental delay was useful for quantification of this effect, it did lump together pups with a one-week delay with those with a more severe delay. The runts from leucine-supplemented litters were frequently further than a week behing normal development. Also, less-severe developmental delays (i.e., less than one week) among siblings were frequently observed in the leucinesupplemented litters and only rarely in the control litters. In the control litters there either was or was not a classical runt with more or less equal development among the other siblings. The quantification therefore underrepresents the effect of leucine supplementation.

There was no difference between control and leucine-supplemented litters in the percentage of pups in the litter that died (Table 1) and there was no correlation

Table 1. Effect of elevated dietary leucine on fertility, litter size and pup survival.

\begin{tabular}{ccccc}
\hline & No litter & Delayed birth $^{\mathrm{a}}$ & Average litter size & Pup deaths $^{\mathrm{d}}$ \\
\hline Control & $4 / 18(22 \%)$ & $3 / 18$ & $8.3^{\mathrm{b}} / 10.6^{\mathrm{c}}$ & $35 / 149(23.5 \%)$ \\
Leucine & $5 / 27(18 \%)$ & $2 / 27$ & $7.6 / 9.4$ & $49 / 207(23.7 \%)$ \\
& NS & NS & NS & NS \\
\hline
\end{tabular}

${ }^{a}$ More than 4 days (one cycle) after the others. ${ }^{\mathrm{b}}$ Average including females with 0 pups. Pups/litter. ${ }^{\mathrm{c}}$ Average not including females with 0 pups. Pups/litter. ${ }^{\mathrm{d}}$ There was no apparent difference in age at death. Expressed as a percentage of live births. NS, no significant difference between control and leucine-supplemented groups. Results compiled from 3 separate trials. 
Table 2. Effect of dietary leucine on pup development.

\begin{tabular}{|c|c|c|c|c|c|c|}
\hline & \multicolumn{3}{|c|}{ Control } & \multicolumn{3}{|c|}{ Leucine } \\
\hline & Pups/litter ${ }^{a}$ & Runts $^{\mathrm{b}}$ & $\%$ Runts & Pups/litter ${ }^{\mathrm{a}}$ & Runts $^{\mathrm{b}}$ & $\%$ Runts \\
\hline & 12 & 1 & & 7 & 1 & \\
\hline & 11 & 0 & & 8 & 1 & \\
\hline & 11 & 0 & & 8 & 1 & \\
\hline & 11 & 1 & & 6 & 1 & \\
\hline & 11 & 0 & & 6 & 1 & \\
\hline & 11 & 0 & & 6 & 1 & \\
\hline & 16 & 0 & & 12 & 1 & \\
\hline & 12 & 1 & & 13 & 1 & \\
\hline & 14 & 1 & & 13 & 1 & \\
\hline & 8 & 0 & & 12 & 0 & \\
\hline & 13 & 0 & & 11 & 1 & \\
\hline & 5 & 0 & & 12 & 0 & \\
\hline & 4 & 0 & & 13 & 1 & \\
\hline & 10 & 1 & & 2 & 1 & \\
\hline & & & & 11 & 0 & \\
\hline & & & & 7 & 0 & \\
\hline & & & & 12 & 1 & \\
\hline & & & & 8 & 0 & \\
\hline & & & & 13 & 0 & \\
\hline & & & & 9 & 1 & \\
\hline & & & & 5 & 0 & \\
\hline & & & & 12 & 1 & \\
\hline Mean & 10.64 & $0.357^{\mathrm{c}}$ & $3.06^{\mathrm{d}}$ & 9.41 & $0.727^{\mathrm{c}}$ & $9.78^{d}$ \\
\hline SEM & 0.856 & 0.133 & 1.15 & 0.695 & 0.09 & 2.28 \\
\hline
\end{tabular}

${ }^{a}$ Using only litters with 1 or more pups. ${ }^{b}$ One or more week's delay in development compared to most advanced siblings. ${ }^{\mathrm{c}}$ Significantly different at $p=0.033$ using 26 degrees of freedom. ${ }^{\mathrm{d}}$ Significantly different at $p=0.013$ using 29.9 degrees of freedom. SEM, standard error of the mean.

between litter size and the presence or absence of a runt in the litter (correlation coefficient 0.28 for controls, 0.015 for leucine-supplemented). An extreme example of this lack of correlation was the litter of 2 pups that had a runt (Table 2).

\section{Consumption of food and water}

No difference was noted in the total amount of food and water consumed by both groups. However, it was obvious that the leucine-supplemented mothers pulled solid food into the cages to feed the pups.

\section{Mammary gland development}

Visual inspection of the mothers showed a clear difference between the control and leucine-supplemented animals. The leucine-supplemented animals had less 

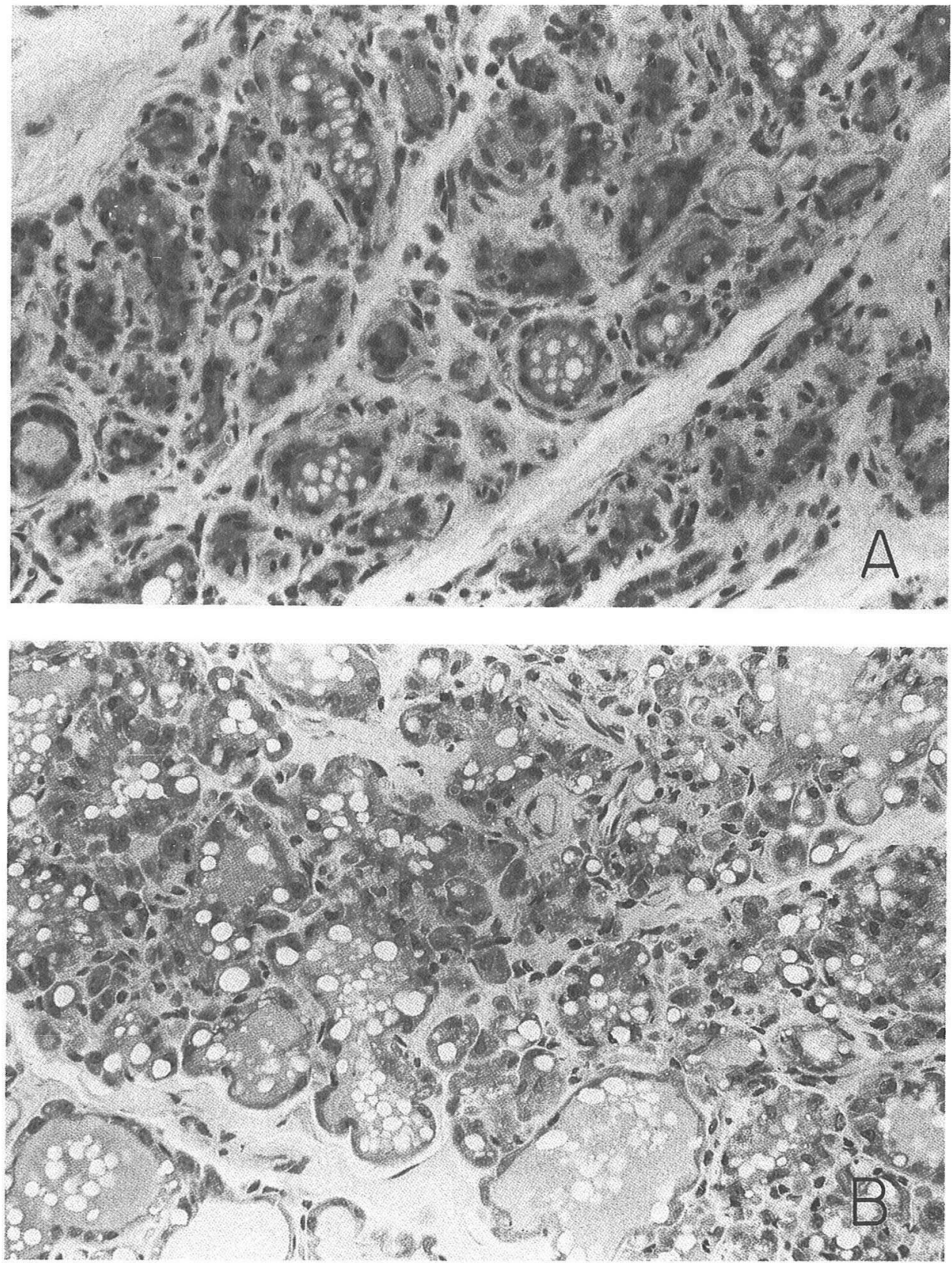

Fig. 1. Mammary tissue from leucine-supplemented (A) and control (B) mothers. Representative photomicrographs of similar areas of equivalent mammary glands. Magnification, $\times 130$. 
obvious mammary tissue. For comparison with control, secretory tissue was removed from glands in the inguinal region where the glands were best developed. The histology of the best-developed glands from the leucine-supplemented mothers was therefore compared with equivalent glands from the control mothers. Mammary tissue from the leucine-supplemented mothers showed normal ductular and less, but normal, alveolar development, and fewer signs of active secretion (compare Fig. 1, A and B) such as lipid droplets and accumulated milk.

\section{DISCUSSION}

Fertility. Previous work from this laboratory (1) and from another laboratory (2) has shown elevated dietary leucine to disrupt the estrous cycle of rats. Our previous study showed the effect of leucine to be an actual interruption of cycling. If the rats were in proestrus when leucine was first administered, then they remained in proestrus. If the rats were in diestrus, then they remained in diestrus. At stages in between, the cycle progressed to diestrus and then stopped. Based on this previous work, a one-week administration of $0.5 \%$ leucine prior to introduction of the males should have ensured that all females were in proestrus or diestrus and not responsive to the males. It was thought likely therefore that leucine administration would have an effect on fertility, but none was observed. It is assumed therefore that something akin to the Whitten effect (male pheromone-induced estrus (5) was a stronger influence on cycle staging than dietary leucine or, alternatively, that the males effectively copulated with females who were not in estrus (6) and that the resultant cervical stimulation was sufficient to overcome the inhibitory effects of leucine. There are conflicting reports in the literature concerning the operation of the Whitten effect in rats $(7,8)$ and our data provide no direct support either for or against the operation of this effect since the protocol used cannot distinguish between pheromone- or cervical stimulation-induced estrus.

Although no effect of leucine on fertility in rats was observed, the possibility of an effect on fertility in other species where estrus is less easily induced deserves investigation since this could be a factor in agricultural production rates or in reduced fertility in societies with a high protein consumption. A single high protein meal can elevate plasma levels of leucine by as much as $600 \%$. It remains to be established, however, that other amino acids in a protein meal do not negate the proposed effects.

Gestation. Dietary supplementation of leucine throughout the gestation period had no effect on the duration and maintenance of pregnancy. There are several possible explanations for this but the most likely in our opinion is that placental lactogens were able to compensate for the alterations in prolactin secretion. It is certainly true in rats that placental lactogens are capable of substituting for pituitary prolactin in the maintenance of pregnancy after day 6 of gestation (9) and that decidual prolactin can substitute during days 2 through 5, even though prolactin itself is required during very early gestation (10-13). 
Pup development and survival. Although we noted no difference in the actual percentage of pups that died in the two groups, a very clear effect on pup development was noted in all three trials. The pups were not weaned until 28 days of age in order for us to follow the delayed development in the pups of leucinesupplemented mothers. Runts occurred in the litters of leucine-supplemented mothers 2-3 times as often as in control litters. A runt was defined as a pup with a week or more's delay in hair growth compared to its most advanced siblings. Not only were there more runts in the leucine litters, but the developmental delay of the runts was usually more severe. The leucine runts were sometimes as much as three weeks behind their siblings. What is surprising about this situation is that it did not lead to an increase in pup mortality. The possible reasons for this are discussed in the next section. In order to gain some insight into whether the effect on pup development was a direct action of leucine on the pups (via its presence in maternal milk) or an effect on maternal lactation, we examined the mammary tissue of the rats in both groups.

Mammary development. As judged by end-point examination of the tissue, there was no major effect on glandular development in the sense that both ductular and alveolar tissue was present, but there was a reduction in alveolar tissue and an effect on the apparent level of activity in the mammary tissue. The grossly observed differences in the amount of mammary tissue between the control and leucinetreated rats could have resulted from a lack of further growth of the glands after parturition (14) and/or from a decreased level of milk production during lactation, the latter being obvious from histological examination of the tissue. Tissue from the control mothers contained large amounts of stored milk and was composed of cells with abundant rough endoplasmic reticulum and lipid droplets. Tissue from the leucine-supplemented mothers contained very little milk, fewer active cells and much more connective tissue. The differences in milk storage cannot be accounted for by the timing of pup feeding as the tissue was obtained the day following pup weaning. Also, late weaning of the pups would likely have decreased any differences between tissues from control and leucine-supplemented mothers because of the normal decrease in secretory activity seen in control rats approaching the time of pup weaning (15). Thus the end point chosen probably underestimates the differences during the period of full lactation in the controls.

That at least a proportion of ductal and alveolar gland development occurred normally suggests that again placental lactogens were capable of fulfilling or supplementing the role of prolactin in mammary gland development. The ability of placental lactogens to supplement for pituitary prolactin during mammary gland development and initial lactation in rats is well documented (for review, see Ref 16) and, at least in humans, secretion of placental lactogens is not affected by the amount of protein in the diet (17). In fact, in rats there is a better correlation between placental weight and the degree of mammary development than there is between pituitary prolactin and growth hormone and mammary development, suggesting that in this species, placental lactogens are of prime importance (18). 
After parturition, however, placental lactogens quite obviously cannot fulfill a lactogenic role and this is when we saw the significant effects of leucine supplementation on the mammary gland. Leucine supplementation could have affected mammary growth and secretion by a variety of means including prolactin secretion (1), growth hormone secretion (19), and insulin secretion (20). Because of the primary importance of prolactin in lactogenesis in the rat (21), however, the most likely interpretation would seem to be via an effect on prolactin, although we cannot exclude the additional or alternative effects of growth hormone and insulin. For both pituitary hormones, leucine supplementation alters the rhythm of secretion, but has no effect on the overall amount of hormone secreted $(1,19)$. This interestingly implies that the rhythm of anterior pituitary hormone release and not only the amount of hormone is important for the maintenance of lactation.

Although mammary gland morphology and underdevelopment of pups implied that milk production was impaired in the leucine-supplemented mothers, milk production was sufficient to ensure the survival of the same average number of pups as the control mothers. Lactational performance was not assessed by pup weights before and after feeding because disturbance of the litters by removal of pups for several hours was judged likely to affect runt survival and hence the results obtained. In part, survival of the leucine pups was ensured by early weaning or partial weaning as the mothers pulled solid food into the cages and young pups were observed at least attempting to eat it. The pups were clearly unable to reach the solid food themselves. This behavior of the leucine-supplemented mothers represents an unusual, definitely unexpected, and to our knowledge previously undocumented, level of maternal nurturing for rats. Unfortunately, we are not in a position to repeat these experiments with caging designed to prevent this occurrence. From our observations, however, we would predict an even larger number of runts and probably also significantly more pup deaths for the leucinesupplemented animals if solid supplementation were eliminated. Early weaning in response to low milk availability has been reported by Thiels and Alberts (22) in an experiment where solid food and water was made available to milk-poor pups.

Although one might expect a positive correlation between the presence of a runt in a litter and litter size, no such correlation was seen in our experiment either in the control or leucine-supplemented rats. We suggest that this is due to the positive correlation between mammary gland development and the number of pups (18). In other words the larger the number of pups, the greater the amount of secretory tissue at the beginning of lactation. Runt occurrence would therefore be more dependent on lactational activity after parturition and/or inherent problems in the pups themselves and this is what we observed.

Although, because of the alterations in mammary gland histology, we have focused the discussion on the likelihood that pup development problems were secondary to lactational problems, we cannot exclude the possibility that excess leucine in maternal milk directly affected the pups through alterations in prolactin, growth hormone or insulin secretion. Whatever the mechanism, however, it is 
interesting that a mild dietary alteration can have such a distinct influence on pup development. If shown to occur, similar effects on apparent mammary gland activity and offspring development in domestic animals could have important agricultural consequences. In humans these dietary influences should be assessed for their persistence when the leucine in derived from a protein meal and therefore for their possible contribution to reduced fertility and lactation in societies that consume large quantities of protein.

The authors would like to thank Sally Scott and Leigh Bruno for ensuring minimal disturbance of the animals during this study, Jane Greenan for expert technical assistance with the histology and photomicrography, Laurie Bollinger for typing the manuscript and Charles Huszar, principal statistician, for discussions concerning appropriate analysis of the data.

This work was supported by NH grants AM 28534 and RR 05816 and by a grant from the University of California, Riverside Academic Senate. TJ was also a recipient of an undergraduate student minigrant.

\section{REFERENCES}

1) Miller, N., Smith, P. A., and Walker A. M. (1986): Increased dietary leucine changes the rhythm of prolactin secretion. Metabolism, 35, 622-626.

2) Cooper, R. L., and Linnoila, M. (1980): Effects of centrally and systemically administered L-tyrosine and L-leucine on ovarian function in the old rat. Gerontology, 26, 270-275.

3) Tannous, R. J., Rogers, Q. R., and Harper, A. E. (1966): Effect of leucine-isoleucine antagonism on the amino acid pattern of plasma and tissues of the rat. Arch. Biochem. Biophys., 113, 356-361.

4) Karnovsky, M. J. (1965): Formaldehyde-glutaraldehyde fixative of high osmolality for use in electron microscopy. J. Cell. Biol., 27, 137E, (abstr).

5) Whitten, W. K. (1956): Modification of the oestrus cycle of the mouse by external stimuli associated with the male. J. Endocrinol., 13, 399-404.

6) Inglis, J. K. (1980): Introduction to Laboratory Animal science and Technology, Pergamon Press, New York, pp. 156-234.

7) May, D. (1969): Synchronization of estrus in the rat. J. Inst. Anim. Technol., 20, 155161.

8) Ritchie, D. H., and Humphrey, J. K. (1970): Some observations on the mating of rats. J. Inst. Anim. Technol., 21, 100-105.

9) Blank, M. S., Chan, J. S., and Friesen, H. G. (1977): Placental lactogens, new developments. J. Steroid Biochem., 8, 403-414.

10) Nicoll, C. S. (1974): Physiological actions of prolactin, in Handbook of Physiology, American Physiol Soc., Washington, Vol. 4, pp. 253-292.

11) Gibori, G., Rothchild, I., Pepe, G., Morishige, W. K., and Lam, P. (1974): Luteotrophic action of decidual tissue in the rat. Endocrinology, 95, 1113-1118.

12) Castracane, V. D., and Rothchild, I. (1976): Luteotrophic action of decidual tissue in the rat: Comparison of jugular and uterine vein progesterone levels, and the effect of

Vol. 36, No. 6, 1990 
ligation of the utero-ovarian connections. Biol. Reprod., 15, 497-503.

13) Basuray, R., and Gibori, G. (1980): Luteotropic action of decidual tissue in the pregnant rat. Biol. Reprod., 23, 507-512.

14) Tucker, H. A., and Reece, R. P. (1963): Nucleic acid content of mammary glands of lactating rats. Proc. Soc. Exp. Biol. Med., 112, 409-412.

15) Hollmann, K. H. (1974): Development and structure of the mammary gland, in Lactation, ed. by Larson, B. L., and Smith, V. R., Academic Press, New York, pp. 391.

16) Talamantes, F., Ogren, L., Markoff, E., Woodard, S., and Madrir, J. (1980): Phylogenetic distribution, regulation of secretion, and prolactin-like effects placental lactogens. Fed Proc., 39, 2582-2587.

17) Tyson, J. E., Jones, G. S., Huth, J., and Thomas, P. (1971): Patterns of insulin, growth hormone, and placental lactogen release after protein and glucose-protein ingestion in pregnancy. Am. J. Obstest. Gynecol., 110, 934-942.

18) Nagasawa, H., and Yanai, R. (1971): Quantitative participation of placental mammotropic hormones in mammary development during pregnancy in mice. Endocrinol. Jpn., 18, 507-510.

19) Stewart, J. K., Koerker, D. J., and Goodner, C. J. (1984): Effects of branched chain amino acids on spontaneous growth hormone secretion in the baboon. Endocrinology, 115, $1897-1900$.

20) Malaisse, W. J., and Malaisse-Lagae, F. (1968): Stimulation of insulin secretion by non-carbohydrate metabolites. J. Lab. Clin. Med., 72, 438-448.

21) Tucker, H. A. (1985): Endocrine and neural control of the mammary gland, in Lactation, ed. by Larson, B. L., Iowa State University Press, Ames, pp. 37-76.

22) Thiels, E., and Alberts, J. R. (1985): Milk availability modulates weaning in the Norway rat. J. Comp. Psychol., 99, 447-456. 